The Reasoned Action Approach Applied to Health Behavior: Role of Past Behavior and Test of Some Key Moderators Using Meta-Analytic Structural Equation Modeling

Martin S. Hagger ${ }^{\mathrm{a}, \mathrm{b}, \mathrm{c}, \mathrm{d}^{* \dagger} \dagger}$, Juho Polet ${ }^{\mathrm{b} \dagger}$, Taru Lintunen ${ }^{\mathrm{b}}$

${ }^{a}$ Health Psychology and Behavioral Medicine Research Group, School of Psychology, Faculty of Health Sciences, Curtin University, Perth, Australia

${ }^{b}$ Faculty of Sport and Health Sciences, University of Jyväskylä, Jyväskylä, Finland

${ }^{\mathrm{c} S}$ School of Applied Psychology, Menzies Health Institute Queensland, Griffith University, Mt.

Gravatt, Australia

${ }^{\mathrm{d}}$ Department of Physical Education, Hong Kong Baptist University, Hong Kong

${ }^{*}$ Correspondence: Martin S. Hagger, Health Psychology and Behavioral Medicine Research Group, School of Psychology, Faculty of Health Sciences, Curtin University, GPO Box U1987, Perth, WA6845, Australia, email: martin.hagger@ curtin.edu.au

${ }^{\dagger}$ These authors contributed equally to the manuscript

Full citation: Hagger, M. S., Polet, J., \& Lintunen, T. (2018). The reasoned action approach applied to health behavior: Role of past behavior and test of some key moderators using metaanalytic structural equation modeling. Social Science \& Medicine. doi:

10.1016/j.socscimed.2018.07.038

\title{
Acknowledgements and Conflict of Interest Statement
}

We thank Mike W. L. Cheung for his advice on the MASEM analysis. Martin S. Hagger's contribution was supported by a Finland Distinguished Professor (FiDiPro) award (Dnro 1801/31/2105) from Business Finland, and a Kennedy Y. H. Wong Distinguished Visiting Professorship from Hong Kong Baptist University. The funders had no role in the study design, the collection, analysis and interpretation of data, writing the article, and in the decision to submit for publication. Martin S. Hagger and Juho Polet contributed equally to this manuscript so it should be considered jointly first-authored. Martin S. Hagger, Juho Polet, and Taru Lintunen declare they have no conflict of interest. 


\begin{abstract}
Rationale: The reasoned action approach (RAA) is a social cognitive model that outlines the determinants of intentional behavior. Primary and meta-analytic studies support RAA predictions for multiple health behaviors. However, including past behavior as a predictor in the RAA may attenuate model effects. Direct effects of past behavior on behavior may reflect non-conscious processes while indirect effects of past behavior through social cognitive variables may represent reasoned processes. Objective: The present study extended a previous meta-analysis of the RAA by including effects of past behavior. The analysis also tested effects of candidate moderators of model predictions: behavioral frequency, behavior type, and measurement lag.
\end{abstract}

Method: We augmented a previous meta-analytic data set with correlations between model constructs and past behavior. We tested RAA models that included and excluded past behavior using metaanalytic structural equation modeling and compared the effects. Separate models were estimated in studies on high and low frequency behaviors, studies on different types of behavior, and studies with longer and shorter measurement lag.

Results: Including past behavior attenuated model effects, particularly the direct effect of intentions on behavior, and indirect effects of experiential attitudes, descriptive norms, and capacity on behavior through intentions. Moderator analyses revealed larger intention-behavior and past behavior-behavior effects in high frequency studies, but the differences were not significant. No other notable moderator effects were observed.

Conclusion: Findings indicate a prominent role for non-conscious processes in determining health behavior and inclusion of past behavior in RAA tests is important to yield precise estimates of model effects.

Keywords: habit; non-conscious processes; theory of planned behavior; dual-process theories; behavioral frequency 


\section{Introduction}

Epidemiological research has consistently identified pervasive links between regular participation in health-related behavior (e.g., physical activity, healthy eating, not smoking, drinking alcohol only in moderation) and reduced risk of chronic disease (e.g., cancers, cardiovascular disease, diabetes) and adaptive health outcomes (Ford et al., 2011; Li et al., 2018). The development of an evidence base of the psychological factors associated with participation in health behaviors has been identified as a priority in behavioral medicine (Conner \& Norman, 2015; Sheeran et al., 2017b). The evidence may inform practice by identifying the salient constructs to target in behavioral interventions (Kok et al., 2016). Theories of social cognition have been at the forefront of research on psychological correlates of health behavior as they provide a fundamental understanding of behavior and the associated processes. Prominent among these theories is the reasoned action approach (RAA; Fishbein \& Ajzen, 2009). The RAA is a generalized, belief-based theory that identifies sets of personal, social, and control-related factors that impact social behavior and the mechanisms involved. McEachan et al.'s (2016) meta-analysis of studies applying the RAA in health behavior contexts provided generalized support for its predictions. In the current article, we extended McEachan et al.'s findings by examining the role of past behavior as an indirect predictor of RAA variables in health behavior contexts. In addition, we tested effects of candidate moderators on RAA relations: behavioral frequency, behavior type, and time lag between measures of RAA constructs and behavior. We expected our analysis to advance previous findings by illustrating that inclusion of past behavior in the RAA provides important information on the extent to which health behavior may be subject to non-conscious processes. In addition, we expected studies on target behaviors that are likely to be performed more frequently, therefore offering greater opportunity for habit formation, to have larger effects of past behavior, and smaller effects of intentions, on behavior. We also expected effects of RAA constructs on behavior to be smaller in groups of studies with longer measurement lag.

\section{The Reasoned Action Approach}


The RAA is a social cognitive model that has been applied to predict and explain behavior in multiple contexts (Fishbein \& Ajzen, 2009). The RAA is a more comprehensive version of its precursor, the theory of planned behavior (TPB; for reviews see Armitage \& Conner, 2001; Hagger et al., 2002; Rich et al., 2015), and distinguishes between specific subcomponents of the TPB constructs. Intention is a central construct of the RAA. It reflects the extent to which individuals are motivated to perform a given behavior and is conceptualized as the most proximal antecedent of behavior. Intentions are a function of three sets of belief-based constructs: attitude, subjective norms, and perceived behavioral control (PBC). Attitudes are an individual's overall positive or negative evaluation of a behavior. The attitude construct is differentiated into experiential and instrumental subcomponents that reflect the affective and utilitarian functions of attitudes, respectively (e.g., Lawton et al., 2009). Subjective norms reflect the individual's beliefs that significant others want them to participate in the behavior. Subjective norms comprise injunctive and descriptive subcomponents (e.g., Sheeran \& Orbell, 1999), which describe social pressures to perform the behavior and beliefs in the extent to which the behavior is typical or normal, respectively. PBC reflects the extent to which the behavior is under the individual's control. PBC is differentiated into autonomy and capacity subcomponents (e.g., Terry \& O'Leary, 1995), which reflect perceptions of control over doing the behavior and perceived confidence in doing it, respectively. Intentions are proposed to mediate relations between the belief-based constructs and behavior.

Predictive validity of the individual subcomponents of the RAA constructs has been supported in primary studies and meta-analytic reviews. Studies distinguishing between instrumental and experiential attitudes indicate that both subcomponents predict intentions directly, but also revealed direct effects of experiential attitudes on behavior. Although not previously hypothesized, the direct effects were interpreted as indicative of more spontaneous, non-conscious effects of affective responses on action (Conner et al., 2015; Lawton et al., 2009). Research has demonstrated significant effects of both normative components on intentions, with effects sizes for descriptive norms tending 
to be larger than those for injunctive norms (Manning, 2009; Rivis \& Sheeran, 2003; Sheeran \& Orbell, 1999). Studies examining effects of capacity and autonomy subcomponents of PBC have demonstrated that capacity tends to be more influential as a predictor of intentions and, directly, behavior (Rodgers et al., 2008; Terry \& O'Leary, 1995). Together these analyses provide converging evidence for the distinction between the subcomponents of the TPB constructs, consistent with hypotheses of the RAA.

Further support for the RAA has been provided in a meta-analysis of prospective studies on health behavior that included RAA subcomponents (McEachan et al., 2016). Regression analyses based on the meta-analysis revealed significant effects for all RAA subcomponents on intentions, with the exception of autonomy. Experiential attitudes and capacity were the most prominent predictors of intentions with small-to-medium effect sizes, with much smaller effects for instrumental attitude and the two normative constructs. Intention significantly predicted health behavior with a medium effect size, with smaller direct effects for descriptive norms, experiential attitudes, and capacity. The authors noted that the direct effects indicate that individuals may be compelled to act spontaneously on the basis of these beliefs without the need for deliberation. Overall, the analysis provided support for the proposed relations among the study constructs.

\section{Importance of Past Behavior}

A prominent omission from McEachan et al.'s meta-analysis was an account for the effects of past behavior in the RAA. Considerable theory and empirical research has focused on the role of past behavior in social cognitive models (e.g., Conner et al., 1999; Hennessy et al., 2010; Ouellette \& Wood, 1998). Past behavior typically exhibits large effects on future behavior, illustrating that behavior tends to have high temporal stability. In the context of social cognitive models, research has consistently shown that the inclusion of past behavior as a predictor of behavior alongside the theorydetermined constructs has four important effects: (a) past behavior predicts behavior; (b) it predicts the other social cognitive variables in the model, including intention; (c) it attenuates effects of the 
other social cognitive variables on intentions and behavior; and (d) it leads to a significant increase in the amount of variance in behavior accounted for by the model (Ajzen, 2002; Brown et al., 2017; Hagger et al., 2016; Hagger et al., 2002; Hamilton et al., 2017; Norman \& Cooper, 2011; Protogerou et al., 2018).

Theorists have suggested that direct effects of past behavior on behavior in social cognitive models reflect implicit or non-conscious processes that affect behavior beyond an individual's awareness (Dombrowski \& Luszczynska, 2009; Hamilton et al., 2017; Wood et al., 2014). This perspective is consistent with dual process theories of cognition and behavior, which predict that participation in health behaviors is a function of two processes: a reasoned, deliberative processes, that tend to be represented by the belief-based constructs in social cognitive theories like the RAA, and an implicit, impulsive process, that can be represented by past behavior (Hagger, 2016; Sheeran et al., 2013; Strack \& Deutsch, 2004). Past behavior effects may model a number of different types of non-conscious processes. For example, Ouellette and Wood (1998) suggest that direct effects of past behavior on behavior independent of effects of other social cognitive constructs may reflect habits. A habit is defined as an action or behavioral tendency that it enacted spontaneously, with little conscious awareness or reflection, in response to a set of associated conditions or contextual cues. Research has indicated that contextual factors implicated in the development of habits, such as opportunity for frequent repetition of the behavior in the presence of a stable environment or facilitating conditions, determine whether behavior is predominantly determined by intentions or past behavior (Ouellette \& Wood, 1998; Wood, 2017). Large effects of past behavior on behavior relative to intentions may be indicative of a habitual action, while larger effects of intentions on behavior relative to past behavior may be indicative of an action controlled by reasoned processes.

Inclusion of past behavior in social cognitive models may also attenuate effects of social cognitive constructs on behavior. This likely reflects previous or 'habitual' decision making; that individuals have processed information about the behavior in a similar way previously. Such previous 
decision making is likely captured by measures of the social cognitive constructs in the RAA, and can be modeled by past behavior. In such cases, variance the social cognitive constructs attributable to previous decision making that is shared with behavior is also shared with past behavior. In the absence of past behavior, effects of these variables may give a misleading indication of the extent to which the behavior is determined by constructs representing reasoned processes that lead to action (Ajzen, 2002). Inclusion of past behavior in social cognitive theories is, therefore, important to provide precise estimates of the unique effects of theory constructs on behavior.

Effects of past behavior may also reflect effects of other unmeasured constructs on behavior that represent implicit, non-conscious determinants of behavior. For example, direct effects of past behavior may reflect effects of implicit cognitions or behavioral scripts, constructs that represent stored sets of information developed over time that initiate behavior beyond an individual's awareness (Abelson, 1981; Ajzen, 2002). These implicit behavioral determinants have proposed to be separable from habits (Ouellette \& Wood, 1998; Wood, 2017). The inclusion of past behavior as a predictor of intentions and behavior in tests of social cognitive models may, therefore, provide important information on the extent to which the behavior is controlled by non-conscious processes.

It is important to note that inclusion of past behavior may diminish the predictive validity of social cognitive models. Constructs in social cognitive models should account for effects of past behavior on subsequent behavior, that is, behavioral stability. However, if past behavior completely accounts for the effects of the social cognitive models on behavior, or renders them relatively trivial by comparison, then model constructs are effectively redundant as a means to explain behavior. It also has serious implications for the effectiveness of the model as a means to inform interventions aimed at changing behavior. If the social cognitive constructs assumed to be manipulable through intervention do not predict behavior, then affecting change in the constructs using intervention techniques will have no concomitant effect on behavior. In cases where past behavior is the dominant predictor and social cognitive constructs account for very little variance in behavior, it may be 
necessary to look to constructs unaccounted for in traditional social cognitive models, such as habit (van Bree et al., 2017; van Bree et al., 2015), implicit attitudes (Gawronski, 2018), implicit motives (Keatley et al., 2012), or behavioral scripts (Abelson, 1981), as candidate mediators of past behavior effects. In such cases, those designing interventions for behavior change might look to identify strategies that target change in these determinants, such as altering the context in which the behavior frequently occurs or avoiding cues that activate implicit cognitions (Hagger, 2016; Hollands et al., 2016). In summary, accounting for past behavior may provide important information on the extent to which behavior is under the control of reasoned and non-conscious processes. It will also permit evaluation of the sufficiency of the model as an effective means to explain behavior.

\section{The Present Study}

The purpose of the present study was to extend McEachan et al.'s (2016) meta-analysis of the RAA in health behaviors to include past behavior as a precursor of model constructs (Hennessy et al., 2010). Given the pervasive effects that past behavior has on future behavior in tests of social cognitive models, and its attenuating effects of social cognitive constructs on behavior, the omission of past behavior in model tests may lead to the drawing of erroneous conclusions over the size of the effects among the model constructs and, as a consequence, its validity in accounting for variance in behavior. Including past behavior will also provide an indication of the extent to which the behavior is accounted for by non-conscious processes, one of the functions indicated by the past behaviorbehavior relation. In addition, the inclusion of past behavior permitted evaluation of the extent to which the social cognitive variables in the RAA account for the effects of past behavior. Confirmation that the RAA variables account for effects of past behavior on future behavior, would provide support for the sufficiency of the model in accounting for behavioral stability and the reasoned processes that lead to health behavior. This has implications for practice as it will indicate which, if any, RAA constructs should be targeted in health behavior change interventions. 
Specifically, we aimed to extend McEachan et al.'s meta-analysis by augmenting their original correlation matrix among RAA constructs to include past behavior. Next, we tested the RAA model that excludes past behavior (see Figure 1) and compare model effects with the augmented model that includes past behavior (see Figure 2) using meta-analytic structural equation modeling. Overall, we expected our analysis to provide new data on whether RAA effects identified in McEachan et al.'s meta-analysis hold in the presence of past behavior, and provide insight into the process by which past behavior relates to subsequent behavior in health behavior contexts.

We also aimed to test effects of three candidate moderators of RAA model effects within the meta-analysis: behavioral frequency, behavior type, and time lag between social cognitive and behavioral measures. Specifically, we aimed to test whether frequency of performance of the target behavior moderated the effects of intentions on behavior, and the effects of past behavior on intentions and the other model constructs. Consistent with previous theory and research on the role of past behavior in social cognitive models (Ouellette \& Wood, 1998; Wood, 2017), we hypothesized that behaviors affording greater opportunity to be performed frequently (e.g., physical activity, eating healthily) are more likely to be acquired as habits, and, as a consequence, the behavior is more likely to be controlled by non-conscious, automatic processes. In contrast, behaviors that are likely to be performed less frequently (e.g., blood donation, screening attendance) offer fewer opportunities for habit formation, and actions are, therefore, more likely to be determined by intentional, reasoned processes. We therefore predicted that the effect of intentions on behavior would be smaller, and the effect of past behavior on behavior as a proxy for habit, would be larger, in studies on behaviors that tend to be performed frequently. It is important to note that behaviors are likely to be habitually controlled not only when individuals have high opportunity to perform them frequently, but also when they are performed in stable contexts (Ouellette \& Wood, 1998). Although frequency and context stability are often associated, segregating behaviors in terms of their opportunity to be 
performed frequently without considering context stability is a relatively crude means to identify conditions that influence habitual control over behavior.

We also aimed to test whether model effects varied across different behaviors. Following McEachan et al.'s (2016) original analysis, we tested whether RAA predictions were consistent across studies with target behaviors that offer protection from risks to health (e.g., physical activity, healthy eating) and studies on target behaviors that are risky for health (e.g., smoking, drinking alcohol). Although they did not make specific predictions, McEachan et al. suggested that experiential attitudes were more likely to have larger effects on risk behavior when compared to protection behaviors given that participation in these behaviors might be determined by affective-related beliefs than instrumental beliefs. Their analysis supported this prediction with larger effects for experiential attitudes, but they also found larger effects for instrumental attitudes and descriptive norms. McEachan et al.'s moderator analyses were confined to univariate relations between model constructs. We aimed to extend this analysis by examining moderator effects in a full test of the RAA in which all model paths are estimated simultaneously. In addition, some behaviors, such as physical activity and dietary-related behaviors, were frequently tested in the set of studies used by McEachan et al., so we aimed to test whether or not model effects were consistent across specific behaviors.

McEachan et al. also tested effects of time lag between measures of RAA model constructs and behavior on individual correlations between the constructs. We aimed to conduct a similar analysis, but in the context of the full structural model. Consistent with previous meta-analytic research adopting social cognitive models indicating that behavioral prediction over time tends lead to smaller effects (Hagger et al., 2002; Mankarious \& Kothe, 2015; McEachan et al., 2011), we expected effects of social cognitive factors, intentions, and past behavior on behavior to be larger when behavior is measured in close proximity to the social cognitive variables. However, we did not expect the RAA to be entropic, that is, for the proposed model effects to wane over time to zero, partly because temporal correspondence in measures tends to maintain prediction, but also because there are 
likely other constructs that tend to maintain intentions over time, albeit those that are typically unmeasured, such as implicit motives and attitudes, planning, and self-monitoring.

\section{Method}

Our analysis used the original studies included in McEachan et al.'s (2016) meta-analysis of the RAA for health behaviors. McEachan et al. identified 74 articles reporting 86 separate datasets (total $N=21,245$ ) on the reasoned action approach applied to health behaviors defined as those likely to impact health outcomes including health promoting behaviors (e.g., physical activity, safer sex, and screening attendance) and health compromising behaviors (e.g., drug use). A list of included in the analysis is provided in Appendix A. Our first task was to fill gaps in McEachan et al.'s correlation matrices among RAA constructs variables in each study, particularly relations among RAA constructs and past behavior. We therefore sourced all of the original studies identified in McEachan et al.'s analysis and replicated their procedures for extraction and coding of study data. The RAA constructs in each study were exclusively measured using standardized measures derived from published guidelines (Fishbein \& Ajzen, 2009). The homogeneity in measurement and conceptualization of constructs obviated the need to code the measures for equivalence across studies (Hagger, 2014). Effect sizes (Pearson correlation coefficients; $r$ ) and associated sample sizes for relations among the RAA constructs were extracted from the zero-order intercorrelation matrices of the source studies. We extracted correlations among the RAA measures and past behavior measures where available $(k=$ 33). We also extracted correlations among the experiential and instrumental attitude, injunctive and descriptive norms, and autonomy and capacity constructs which were not reported in McEachan et al.'s original analysis. We aggregated effect sizes at the study level in cases where studies included multiple measures of related behavior measures. When a study measured behavior at multiple followup timepoints, we used the latest timepoint. We also extracted the time lag between measures of RAA constructs and behavior measures, for each study. Our final sample sizes for each RAA effect was closely matched with McEachan et al.'s original analysis although there were some variations. For 
example, Blanchard et al. (2008) reported data from four samples defined by ethnicity (Caucasian, African-American) and length of follow-up (two-week, two-month), we included all four samples in our analysis while McEachan et al. included only the two-month samples. Collection of studies and data extraction were conducted between May and August 2017.

Next, we tested relations among RAA constructs using meta-analytic structural equation modeling (MASEM) using the metaSEM package (Cheung, 2015) in R (R Development Core Team, 2017). A typical method used to analyze multiple relations among constructs in social cognitive models is to adopt a univariate approach that involves first correcting correlations among variables in the model across studies using meta-analysis and then using the average bias-corrected correlation matrix as input in a multiple regression or path analysis testing the model. This method has been used in many previous studies (e.g., Hagger et al., 2016; Hagger et al., 2017b; Ng et al., 2012) including McEachan et al. (2016) in their analysis of the RAA. However, this procedure has been identified as problematic because it necessitates finding a common sample size to estimate the standard errors, such as the harmonic mean of the sample size across studies, and assumes that the correlation matrix is a covariance matrix, which likely leads to inaccuracies in the resulting standard errors, confidence intervals, and chi-square values of the model (Cheung, 2015). MASEM offers an alternative that directly addresses the inherent limitations of the univariate approach. The analysis is conducted in two stages. In the first stage, correlation matrices from individual studies are pooled to form a common correlation matrix using random effects meta-analysis. The analysis produces a pooled correlation matrix representing the average correlation matrix among study variables in the population, with a sampling variance-covariance matrix that represents the known precision estimates of each correlation in the pooled matrix. The first stage yields zero-order correlations corrected for bias using a random effects meta-analytic model $\left(r^{+} \mathrm{RE}\right)$ among study constructs across studies with 95\% confidence intervals. In addition, statistics to evaluate heterogeneity in the effect sizes are also provided: the $\tau^{2}$ statistic, the $Q$ statistic, and the $I^{2}$ statistic and its $95 \%$ confidence interval. 
Statistically significant $\tau^{2}$ and $Q$ values with $I^{2}$ values exceeding $25 \%$ with wide confidence intervals are considered indicative of substantive heterogeneity. We also computed conventional fixed- and random-effects meta-analytic estimates for each RAA effect using the metafor package in R for comparison.

In the second stage of the analysis, the pooled correlation matrix is used to estimate the proposed model. Two models were tested: a model testing the hypothesized pattern of effects among study constructs consistent with hypotheses from the RAA (see Figure 1) and a model augmented to include past behavior (see Figure 2). Fit of the proposed model with data from the meta-analysis was evaluated using multiple goodness-of-fit indices: the model goodness-of-fit chi-square, the comparative fit index (CFI), the Tucker-Lewis index (TLI), the standardized root mean square of the residuals, and the root mean error of approximation (RMSEA). A non-significant chi-square, CFI and TLI values that approach or exceed .95 , a SRMSR value of less than .008 , and a RMSEA value of .005 or less, indicate good fit of the model with the data (Hu \& Bentler, 1999). Effects among model constructs were evaluated based on the likelihood based confidence intervals about model parameter estimates. We evaluated whether inclusion of past behavior in the model attenuated effects of RAA constructs on intentions, and of intentions on behavior, by computing the confidence interval of the difference in the parameter estimates across the models (Schenker \& Gentleman, 2001). To the extent that the interval does not include zero, we have confirmation of a statistically significant difference in the parameter estimates across models.

We evaluated effects of behavior type, and time lag moderators on RAA model effects by estimating the models separately in groups of studies on behaviors classified as high and low frequency, studies on specific behaviors that had been tested with high regularity, studies on health risk and health protection behaviors, and studies with longer and shorter time lag between social cognitive and behavioral measures. Classification of studies into high $(k=65)$ and low $(k=16)$ frequency was based on Ouellette and Wood's (1998) criterion. Although classification of studies into 
those targeting behaviors that offered greater opportunity to be performed with high (e.g., physical activity, health eating) or low frequency (e.g., blood donation, health screening) was a relatively straightforward process, there were particular cases that presented some difficulty. For example, for condom use, individuals in a regular, long-term relationship using condom use for contraception are more likely to have greater opportunity for use of condoms than individuals not in a relationship. Similarly, for speeding when driving, opportunity may be dependent on whether or not the individual used a car on a daily basis, such as commuting to work. In these cases we relied on the definition and operationalization of the behavior and the sample description provided by in the original report as a basis for our decision. Coding for this moderator was conducted independently by two raters. Interrater analysis indicated strong agreement $(\kappa=.85)$. Discrepancies were discussed before settling on a final classification. We also identified groups of studies that tested the RAA in the same target behavior. The most frequently occurring target behaviors in the sample of studies were physical activity $(k=38)$ and dietary behaviors $(k=13)$, with other behaviors adopted with lower frequency $(k$ <10). We retained McEachan et al.'s (2016) original coding of studies into health protection $(k=70)$ and health risk $(k=11)$ behaviors. Consistent with previous meta-analytic studies (e.g., Hagger et al., 2002) including McEachan et al. (2016), studies were classified as having longer measurement lag if the time gap between measurement of the RAA constructs and behavior was greater than 4 weeks $(k=$ $43)$, and shorter if the time lag was four weeks or fewer $(k=38)$. Heterogeneity statistics from the first stage of the MASEM analysis were used to evaluate whether the moderator had resolved the heterogeneity in correlations among variables. Differences in relations among model constructs at the levels of the moderator were tested using the confidence intervals about the difference in model parameter estimates (Schenker \& Gentleman, 2001).

We also evaluated the potential effect of selective reporting bias in relations among the RAA model constructs across included studies using regression analyses based on 'funnel' plots of effect size on estimates of precision. The plots are used to estimate the extent to which an averaged effect 
size derived from a meta-analysis may deviate from the true effect due to selective biases in the sample of studies in the analysis. A principal source of bias may be 'publication bias' caused by a preponderance of studies in the sample with disproportionately large effect sizes relative to the sample size. Regressing effect size on study precision provides an estimate of the extent of bias and an estimate of the effect size corrected for bias. Two methods are used: the precision effect test (PET) and the precision effect estimate with standard error (PEESE). The PET is the linear regression of study effect size on its standard error estimate, weighted by the inverse variance. The intercept of the model provides an unbiased estimate of the true mean effect size. However, the PET may underestimate the true mean effect size when there is evidence of a non-zero effect. The intercept derived from a non-linear (quadratic) regression of study effect size on the square of its standard error, again weighted by the inverse variance, the PEESE, has been shown to provide a more precise estimate of the true mean effect in cases where there is evidence of a non-zero effect. We computed PET and PEESE estimates, with $t$-test for bias, and statistical significance of the bias-corrected effect from zero to provide an indication of selective bias in each estimate using the PETPEESE function in R (Carter et al., 2017). A full table including study characteristics, extracted data, and moderator coding can be accessed online from the Open Science Framework (OSF) project for this study: https://osf.io/w4hfr.

\section{Results}

\section{Zero-order parameter estimates and bias statistics}

Zero-order averaged bias-corrected correlations $\left(r^{+} \mathrm{RE}\right)$ among RAA model constructs including past behavior from the first stage of the MASEM analysis are presented in Appendix B (supplemental materials), with bias-corrected correlations from conventional random- and fixed-effects metaanalysis included for comparison. The bias-corrected correlations from the MASEM analysis were all significantly different from zero and non-trivial in size $\left(r^{+} \mathrm{RE}\right.$ range $=.158$ to .564$)$. Heterogeneity statistics revealed moderate-to-high heterogeneity according to the $I^{2}$ statistic. Values for the $Q$ - 
statistic across studies also indicated substantial heterogeneity for the models excluding $(Q(1244)=$ $6742.459, p<.001)$ and including $(Q(1440)=7666.848, p<.001)$ past behavior. As correlations from the MASEM analysis and conventional meta-analytic models indicated positive non-zero effects, the PEESE regression test was taken as an estimate of selection bias. The test revealed significant bias in the majority of the effects. However, the bias-corrected PEESE estimates did not alter conclusions with respect to whether effects were different from zero and their overall size. Analysis scripts and output files for the metaSEM analysis, conventional meta-analysis, and PETPEESE analysis with funnel plots can be accessed online from the study OSF project: https://osf.io/w4hfr.

\section{Meta-analytic structural equation models}

Goodness-of-fit statistics indicated that the second stage MASEM models of the RAA that excluded (Figure 1) and included (Figure 2) past behavior exhibited adequate fit with the data (see Appendix C, supplemental materials). Standardized parameter estimates with 95\% likelihood-based confidence intervals from the MASEM analyses for both models are presented in Table 1. Patterns of effects for the model excluding past behavior were consistent with those found by McEachan et al. in their regression analyses, albeit with some variation in the magnitude of the effects. Specifically, we found statistically significant, positive direct effects of experiential and instrumental attitudes, injunctive and descriptive norms, and capacity on intentions. We also found significant and positive direct effects of experiential attitudes, capacity, and intentions on behavior. Effect sizes were small ( $\beta$ $<.150$ ), with the exception of the effects for experiential attitude, descriptive norms, and capacity on intentions, and intentions on behavior. In addition, experiential and instrumental attitudes, injunctive and descriptive norms, and capacity had significant and positive indirect effects on health behavior through intentions, consistent with hypotheses. Indirect effects for instrumental attitudes, and injunctive and descriptive norms were relatively small and trivial $(\beta<|.075|)$ (Seaton et al., 2010), 
while indirect effects for experiential attitude and capacity were more substantive. Overall, RAA constructs accounted for $47.51 \%$ and $24.07 \%$ of the variance in intentions and behavior, respectively.

Including past behavior as a predictor of all variables in the model (Figure 2) revealed statistically significant attenuation of some model relations. Specifically, the direct effect of intention on behavior, and the indirect effects of experiential attitudes, descriptive norms, and capacity on behavior through intentions were significantly smaller. The attenuation of key effects notwithstanding, inclusion of past behavior did not lead to a rejection of the RAA as the overall pattern of effects of the model was supported. Including past behavior as a predictor in the model resulted in a significant increase in the proportion of variance explained in intentions $(53.17 \%)$ and behavior (34.41\%), reflecting the pervasive effect of past behavior in the model. In addition, past behavior also exhibited significant indirect effects on behavior mediated by intentions alone, and mediated by the other social cognitive variables and intentions, on behavior. According to the proportion mediation statistic ( $\mathrm{P}_{\mathrm{M}}$; Ditlevsen et al., 2005), the total indirect effect of past behavior through the social cognitive variables from the RAA and intentions accounted for a small proportion of the total effect of past behavior on behavior $\left(P_{M}=.200\right)$. This means that $20 \%$ of the total effect of past behavior on behavior was accounted for by the indirect effects, and indicated that the direct effect of past behavior on future behavior was the larger effect.

\section{Moderator analyses}

MASEM analyses of the RAA in moderator groups representing behavior frequency, behavior type, and measurement lag, exhibited adequate fit with the data according to multiple criteria (see Appendix C, supplemental materials). Standardized parameter estimates, confidence intervals, and test statistics for each moderator analysis are presented in supplemental materials (Appendixes D-G). For the behavior frequency moderator (Appendix D), the direct effects of intention on behavior was larger, and the direct effect of past behavior on behavior smaller, in groups of studies with high frequency, compared to studies with low frequency. These effects were contrary to predictions. 
However, it is important to note that these observed differences were not statistically significant across moderator groups. In addition, we found significantly larger effects of past behavior on experiential attitudes, injunctive norm, and descriptive norm in studies on low frequency behaviors. There were also trends toward larger effects of past behavior on autonomy and capacity in studies on low frequency behaviors.

For the behavior type moderators, the models estimated on dietary behaviors and health risk behaviors did not converge due to small numbers of studies and positive non-definite covariance matrices. The model on health protection behaviors was therefore compared to the full sample, effectively a sensitivity analysis testing whether our conclusions on model effects would change with the exclusion of studies on health risk behaviors. The model estimated on studies on physical activity behaviors was compared to models on other behaviors. We found no differences in model effects for studies on health protection behaviors relative to the overall sample (Appendix E). Effects of past behavior on experiential and instrumental attitudes, and injunctive norms were smaller in studies on physical activity behavior relative to studies on other behaviors. However, there were significant differences or observed trends in the total effects of past behavior and intention on behavior across behavior type (Appendix F). Finally, there were no significant differences or observed trends in model effects across the measurement lag moderator (Appendix G). It seems that model effects were consistent regardless of time gap between measures, indicating that the model effects were consistent in studies with shorter- and longer-term behavioral follow-up.

\section{Discussion}

The purpose of the present study was to extend McEachan et al.'s (2016) meta-analysis of the RAA for health behaviors by including past behavior as a predictor in the model. The analysis aimed to evaluate the extent to which past behavior predicts prospectively-measured health behavior beyond the RAA constructs - effects that likely model habitual, non-conscious processes on health behavior. We also set out to examine the effects of candidate moderators of RAA model effects: behavior 
frequency, behavior type, and time lag between measurement of social cognitive constructs and behavior. We updated McEachan et al.'s database of correlations among the RAA constructs with correlations between these constructs and past behavior, and extracted data on behavioral frequency, behavior type, and measurement lag. MASEM was used to test the proposed RAA models excluding and including past behavior across studies. Results for the model excluding past behavior replicated the pattern of effects reported in McEachan et al.'s original analysis. Inclusion of past behavior resulted in significant attenuation of the direct effect of intentions on behavior, and the indirect effects of experiential attitudes, descriptive norms, and capacity on behavior through intentions. The indirect effects of past behavior through the social cognitive variables in the model and intentions accounted for a relatively modest proportion of the total effect of past behavior on behavior. We found few statistically significant differences or observed trends in model effects when testing the model in studies across levels of the moderator variables.

Current findings point to the pervasive effect of past behavior as a determinant of health behavior, which has two important ramifications for the interpretation of RAA predictions. First, it means that regardless of any variability in effect sizes due to potential moderators (e.g., context, behavior, or population), the observed effects among RAA constructs in health behaviors are likely to be non-zero. Furthermore, the effect of intentions on behavior is likely to be lower when past behavior is included in the model. In other words, researchers testing the model would expect to find these patterns of relations regardless of variations in study characteristics. This is consistent with previous meta-analytic research examining past behavior effects in social cognitive models (Hagger et al., 2016; Hagger et al., 2002), and recent research applying the RAA to health behaviors (Conner et al., 2017). Second, an important implication of these findings is that tests of the RAA in the absence of past behavior are likely to yield inflated estimates of the averaged effects of intention on health behavior, and indirect effects of RAA constructs acting through intentions, and may lead to misleading conclusions regarding the size of the effects. This has important ramifications for the 
RAA given that the intention-behavior relationship is central to the model and fundamental to its validity (Hagger et al., 2017a; Trafimow, 2009). At the aggregate level, our analysis has important implications for interpreting the pattern of effects in the RAA and for future research. Researchers should be encouraged to consider the effects of past behavior in future tests of the RAA, and consider past behavior when interpreting model effects.

However, it is important to note that while the size of these effects among RAA model variables was substantially reduced with the inclusion of past behavior, the effects were not extinguished. Taken together with results from primary studies indicating pervasive effects for past behavior in social cognitive models (e.g., Brown et al., 2017; Hamilton et al., 2017; Norman \& Cooper, 2011), our findings are consistent with explanations offered by dual process theories in which two pathways to action are proposed: a reasoned route, modeled by the effects of the belief-based constructs from the RAA mediated by intentions, and a non-conscious, habitual route, represented by the effects of past behavior (Hagger, 2016; Sheeran et al., 2013; Strack \& Deutsch, 2004). Rather than both processes operating simultaneously, a likely interpretation of the coexistence of these two pathways is that health behavior may be predominantly controlled by one of the processes, determined by moderator variables such as type of population or behavior.

A possible explanation for the past behavior effects in the current synthesis of the RAA is that they model implicit, non-conscious processes on health behavior. To speculate, past behavior effects may reflect the influence of habits. However, Ouellette and Wood's (1998) contention that habitual behaviors tend to be those that individuals have high opportunity to perform in stable contexts, was not supported in our behavior frequency moderator analysis. In fact, we found non-significant trends of smaller effects of past behavior, and larger effects of intentions, on behavior in studies on high frequency behaviors, findings which deviate from our predictions. An explanation may lie in a recent analysis demonstrating that the intention-behavior relationship is curvilinear under the moderating effects of past behavior (Sheeran et al., 2017a). Sheeran et al.'s findings suggest that past behavior 
has a stabilizing effect on the intention-behavior relationship at moderate levels of behavioral frequency. However, as behavioral frequency increases, individuals are more likely to form habits and the role of intentions is diminished. Our results may, therefore, be indicative of the stabilizing effect. We stress that this is a speculative interpretation, and current data did not permit us to verify whether the intention-behavior relation follows a quadratic relation with past behavioral frequency. It is also important to note that these interpretations are based on the strength of the effects alone and must be interpreted in light of a lack of formal differences.

Alternatively, the effects for past behavior effects in the RAA observed in the present study may reflect the influence of behavioral 'scripts'; organized sets of information stored schematically linking the action with cues and contexts built up through repeated past experience (Abelson, 1981). Implicit measures of social cognitive constructs, such as implicit association tests and tasks measuring accessibility of constructs, may provide a means to directly tap beliefs representing this 'scripted' information (Gawronski, 2018; van Bree et al., 2015). Testing whether implicit measures mediate effects of past behavior on prospectively-measured health behavior would provide evidence to corroborate the contention that past behavior effects reflect automatic, non-conscious processes, and is an important avenue for future research.

\section{Study Limitations and Implications for Practice}

A number of limitations that affect inferences and generalizability of current findings should be noted. Studies included in the current analysis were exclusively correlational in design. Such designs do not permit the inference of causal relations among variables in the model. Such inferences are based on theory alone and alternative models that are plausible empirically, if not theoretically, could be proposed (Hagger \& Chatzisarantis, 2016; Hagger et al., 2017a). Studies that test premises of the RAA using cross-lagged panel designs, in which study constructs including behavior are measured at multiple time points, are needed. Panel designs would permit prediction of variable change over time and provide tests of directional and reciprocal relations among constructs, particularly prediction of 
future behavior from intentions and past behavior. Of course such designs are also correlational, so a further alternative would be experimental and correlational designs which manipulate key variables in the RAA and test effects of subsequent intentions and behavior while controlling for past behavior. Another alternative would be to use a quasi-experimental design in which previous experience with the behavior is varied, for example by testing the RAA in novice and experienced participants enroled in a fitness center or exercise program. A further limitation was that most studies relied on self-report measures of past and prospective behavior. This is likely to introduce additional error variance into the relations in the current synthesis due to limits on individuals' ability to recall their behavior, as well as a potential for selective reporting and socially-desirable responding. In addition, self-report measures of past and future behavior usually adopt the same format, and this similarity in measurement is likely to inflate relations due to common-method variance. Research that adopts objective direct or proxy measures for behavior should be advocated to minimize bias. Another limitation was the potential for a lack of correspondence between the RAA measures and behavioral measures. RAA measures frequently tap beliefs with respect to behavioral engagement as a goal or endpoint, while behavior is often measured using frequency measures. This lack of correspondence may introduce method variance to the effects (Ajzen, 1991). We recommend research testing the moderation of model effects by the degree of correspondence between RAA constructs and frequency behavioral measures, and tests that compare model effects using frequency behavioral measures with other behavioral measures such as recency and frequency in context. Finally, effects among RAA constructs in the present analysis were highly variable with wide confidence intervals and substantive heterogeneity. Caution should be taken when drawing conclusions on patterns of effects in the RAA in the presence of high levels of heterogeneity.

Current findings have important implications for interventions to promote and change health behavior. One of the reported advantages of the RAA is that it allows greater precision in identifying the factors most influential in predicting health behavior. Greater precision means greater specificity 
in identifying salient targets for intervention and the behavior change methods to modify them. Differentiation of constructs in the RAA seems to be important given that some factors (e.g., experiential attitudes, capacity) have larger effects on behavior. These factors make viable targets for intervention. However, attenuation of the effects of these variables on behavior through intentions when past behavior was included in the model meant that the potential impact of interventions targeting RAA constructs might be muted. However, knowledge of the pervasive effects of past behavior may also inform interventions. While the effects of past behavior do not highlight a specific target for intervention, it indicates the need for self-regulatory skills which may minimize or circumvent habitual or non-conscious enactment of behaviors. Such skills may include cue identification and management, and depending on the behavior, self-monitoring to manage potential opportunities to act or minimize the threat of lapsing (Kleinjan et al., 2012; Marteau et al., 2012; Miller \& Thayer, 1988).

\section{Conclusion}

Extending McEachan et al.'s (2016) meta-analytic test of the RAA to include past behavior has provided evidence that the RAA may not provide a comprehensive account of health behavior beyond previous experience. Our findings indicate that past behavior predicts prospective health behavior directly, and indirectly, through the social cognitive constructs in the model, and attenuates the effects of intention and social cognitive factors on health behavior. The current evidence seems to point for a substantive role for habitual, automatic processes, represented by the direct effects of past behavior on health behavior. Current results do not, however, invalidate the RAA, the social cognitive constructs in the model remain statistically significant albeit modest predictors. Although current research indicates a possible role of behavioral frequency as a moderator of intention and past behavior effects in the RAA, testing the moderating effects of other variables that likely determine whether behaviors are controlled by habitual or reasoned processes (e.g., behavioral recency, self-reported habit) on past behavior-behavior and intention-behavior relations should be a priority for future research. 


\section{References}

Abelson, R.P. (1981). Psychological status of the script concept. Am. Psychol., 36, 715-729. Ajzen, I. (1991). The theory of planned behavior. Organ. Behav. Hum. Decis. Process., 50, 179-211. Ajzen, I. (2002). Residual effects of past on later behavior: Habituation and reasoned action perspectives. Pers. Soc. Psychol. Rev., 6, 107-122.

Armitage, C.J., \& Conner, M. (2001). Efficacy of the theory of planned behaviour: A meta-analytic review. Br. J. Soc. Psychol., 40, 471-499.

Blanchard, C.M., Kupperman, J., Sparling, P., Nehld, E., Rhodes, R.E., Courneya, K.S., et al. (2008). Ethnicity and the theory of planned behavior in an exercise context: A mediation and moderation perspective. Psychol. Sport Exerc., 9, 527-545.

Brown, D.J., Hagger, M.S., Morrissey, S., \& Hamilton, K. (2017). Predicting fruit and vegetable consumption in long-haul heavy goods vehicle drivers: Application of a multi-theory, dualphase model and the contribution of past behaviour. Appetite, 121, 326-336.

Carter, E.C., Schonbrodt, F., Gervais, W., \& Hilgard, J. (2017). Correcting for bias in psychology: A comparison of meta-analytic methods. Retrived October 10 from https://psyarxiv.com/9h3nu/

Cheung, M.W.L. (2015). metaSEM: an R package for meta-analysis using structural equation modeling. Front. Psychol., 5, 1521.

Conner, M.T., McEachan, R., Lawton, R., \& Gardner, P. (2017). Applying the reasoned action approach to understanding health protection and health risk behaviors. Soc. Sci. Med., 195, 140148.

Conner, M.T., McEachan, R., Taylor, N., O'Hara, J., \& Lawton, R. (2015). Role of affective attitudes and anticipated affective reactions in predicting health behaviors. Health Psychol., 34, 642-652.

Conner, M.T., \& Norman, P. (2015). Predicting and changing health behaviour: Research and practice with social cognition models. Maidenhead, UK: Open University Press.

Conner, M.T., Warren, R., Close, S., \& Sparks, P. (1999). Alcohol consumption and the theory of planned behavior: An examination of the cognitive mediation of past behavior. J. Appl. Soc. Psychol., 29, 1676-1704.

Ditlevsen, S., Christensen, U., Lynch, J., Damsgaard, M.T., \& Keiding, N. (2005). The mediation proportion: A structural equation approach for estimating the proportion of exposure effect on outcome explained by an intermediate variable. Epidemiology, 16, 114-120.

Dombrowski, S., \& Luszczynska, A. (2009). The interplay between conscious and automatic selfregulation and adolescents' physical activity: The role of planning, intentions, and lack of awareness. Appl. Psychol., 58, 257-273.

Fishbein, M., \& Ajzen, I. (2009). Predicting and changing behavior: The reasoned action approach. New York, NY: Psychology Press.

Ford, E.S., Zhao, G.X., Tsai, J., \& Li, C.Y. (2011). Low-risk lifestyle behaviors and all-cause mortality: Findings from the national health and nutrition examination survey III mortality study. Am. J. Public Health, 101, 1922-1929.

Gawronski, B. (2018). Implicit attitudes. In D. Albarracín, \& B.T. Johnson (Eds.), The Handbook of Attitudes. New York, NY: Psychology Press.

Hagger, M.S. (2014). Avoiding the 'déjà-variable' phenomenon: Social psychology needs more guides to constructs. Front. Psychol., 5, 52.

Hagger, M.S. (2016). Non-conscious processes and dual-process theories in health psychology. Health Psychol. Rev., 10, 375-380.

Hagger, M.S., Chan, D.K.C., Protogerou, C., \& Chatzisarantis, N.L.D. (2016). Using meta-analytic path analysis to test theoretical predictions in health behavior: An illustration based on metaanalyses of the theory of planned behavior. Prev. Med., 89, 154-161.

Hagger, M.S., \& Chatzisarantis, N.L.D. (2016). The trans-contextual model of autonomous motivation in education: Conceptual and empirical issues and meta-analysis. Rev. Educ. Res., 86, 360-407. 
Hagger, M.S., Chatzisarantis, N.L.D., \& Biddle, S.J.H. (2002). A meta-analytic review of the theories of reasoned action and planned behavior in physical activity: Predictive validity and the contribution of additional variables. J. Sport Exerc. Psychol., 24, 3-32.

Hagger, M.S., Gucciardi, D.F., \& Chatzisarantis, N.L.D. (2017a). On nomological validity and auxiliary assumptions: The importance of simultaneously testing effects in social cognitive theories applied to health behavior and some guidelines Front. Psychol., 8, 1933.

Hagger, M.S., Koch, S., Chatzisarantis, N.L.D., \& Orbell, S. (2017b). The common-sense model of self-regulation: Meta-analysis and test of a process model. Psychol. Bull., 143, 1117-1154.

Hamilton, K., Kirkpatrick, A., Rebar, A., \& Hagger, M.S. (2017). Child sun safety: Application of an integrated behavior change model. Health Psychol., 36, 916-926.

Hennessy, M., Bleakley, A., Fishbein, M., Brown, L., DiClemente, R., Romer, D., et al. (2010). Differentiating between precursor and control variables when analyzing reasoned action theories. AIDS Behav., 14, 225-236.

Hollands, G.J., Marteau, T.M., \& Fletcher, P.C. (2016). Non-conscious processes in changing healthrelated behaviour: A conceptual analysis and framework. Health Psychol. Rev., 10, 381-394.

Keatley, D.A., Clarke, D.D., \& Hagger, M.S. (2012). Investigating the predictive validity of implicit and explicit measures of motivation on condom use, physical activity, and healthy eating. Psychol. Health, 27, 550-569.

Kleinjan, M., Strick, M., Lemmers, L., \& Engels, R.C. (2012). The effectiveness of a cue-reminder intervention to reduce adolescents' alcohol use in social contexts. Alcohol Alcohol., 47, 451-457.

Kok, G., Gottlieb, N.H., Peters, G.-J.Y., Mullen, P.D., Parcel, G.S., Ruiter, R.A.C., et al. (2016). A taxonomy of behavior change methods: An intervention mapping approach. Health Psychol. Rev., 10, 297-312.

Lawton, R., Conner, M.T., \& McEachan, R. (2009). Desire or reason: Predicting health behaviors from affective and cognitive attitudes. Health Psychol., 28, 56-65.

Li, Y., Pan, A., Wang, D.D., Liu, X., Dhana, K., Franco, O.H., et al. (2018). Impact of healthy lifestyle factors on life expectancies in the US population. Circulation.

Mankarious, E., \& Kothe, E. (2015). A meta-analysis of the effects of measuring theory of planned behaviour constructs on behaviour within prospective studies. Health Psychol. Rev., 9, 190204.

Manning, M. (2009). The effects of subjective norms on behaviour in the theory of planned behaviour: A meta-analysis. Br. J. Soc. Psychol., 48, 649-705.

Marteau, T.M., Hollands, G.J., \& Fletcher, P.C. (2012). Changing human behavior to prevent disease: The importance of targeting automatic processes. Science, 337, 1492-1495.

McEachan, R.R.C., Conner, M.T., Taylor, N., \& Lawton, R.J. (2011). Prospective prediction of health-related behaviors with the theory of planned behavior: A meta-analysis. Health Psychol. Rev., 5, 97-144.

McEachan, R.R.C., Taylor, N., Harrison, R., Lawton, R., Gardner, P., \& Conner, M.T. (2016). Metaanalysis of the reasoned action approach (RAA) to understanding health behaviors. Ann. Behav. Med., 50, 592-612.

Miller, M.L., \& Thayer, J.F. (1988). On the nature of self-monitoring: Relationships with adjustment and identity. Pers. Soc. Psychol. Bull., 14, 544-553.

Ng, J.Y.Y., Ntoumanis, N., Thøgersen-Ntoumani, C., Deci, E.L., Ryan, R.M., Duda, J.L., et al. (2012). Self-determination theory applied to health contexts. Perspect. Psychol. Sci., 7, 325340.

Norman, P., \& Cooper, Y. (2011). The theory of planned behaviour and breast self-examination: Assessing the impact of past behaviour, context stability and habit strength. Psychol. Health, 26, 1156-1172.

Ouellette, J.A., \& Wood, W. (1998). Habit and intention in everyday life: The multiple processes by which past behavior predicts future behavior. Psychol. Bull., 124, 54-74. 
Protogerou, C., Johnson, B.T., \& Hagger, M.S. (2018). An integrated model of condom use in subSaharan African youth: A meta-analysis. Health Psychol., 37, 586-602.

R Development Core Team (2017). R: A language and environment for statistical computing. Vienna, Austria: R Foundation for Statistical Computing.

Rich, A., Brandes, K., Mullan, B.A., \& Hagger, M.S. (2015). Theory of planned behavior and adherence in chronic illness: A meta-analysis. J. Behav. Med., 38, 673-688.

Rivis, A., \& Sheeran, P. (2003). Descriptive norms as an additional predictor in the theory of planned behaviour: A meta-analysis. Curr. Psychol., 22, 218-233.

Rodgers, W.M., Conner, M.T., \& Murray, T.C. (2008). Distinguishing among perceived control, perceived difficulty, and self-efficacy as determinants of intentions and behaviours. Br. J. Soc. Psychol., 47, 607-630.

Schenker, N., \& Gentleman, J.F. (2001). On judging the significance of differences by examining the overlap between confidence intervals. Am. Stat., 55, 182-186.

Seaton, M., Marsh, H.W., \& Craven, R.G. (2010). Big-fish-little-pond Effect: Generalizability and moderation-Two sides of the same coin. Am. Educ. Res. J., 47, 390-433.

Sheeran, P., Godin, G., Conner, M.T., \& Germain, M. (2017a). Paradoxical effects of experience: Past behavior both strengthens and weakens the intention-behavior relationship. 2, 309-318.

Sheeran, P., Gollwitzer, P.M., \& Bargh, J.A. (2013). Nonconscious processes and health. Health Psychol., 32, 460-473.

Sheeran, P., Klein, W.M.P., \& Rothman, A.J. (2017b). Health behavior change: Moving from observation to intervention. Ann. Rev. Psychol., 68.

Sheeran, P., \& Orbell, S. (1999). Augmenting the theory of planned behavior: Roles for anticipated regret and descriptive norms. J. Appl. Soc. Psychol., 29, 2107-2142.

Strack, F., \& Deutsch, R. (2004). Reflective and impulsive determinants of social behavior. Pers. Soc. Psychol. Rev., 8, 220-247.

Terry, D.J., \& O'Leary, J.E. (1995). The theory of planned behaviour: The effects of perceived behavioural control and self-efficacy. Br. J. Soc. Psychol., 34, 199-220.

Trafimow, D. (2009). The theory of reasoned action: A case study of falsification in psychology. Theory Psychol., 19, 501-518.

van Bree, R.J.H., Bolman, C., Mudde, A.N., van Stralen, M.M., Peels, D.A., de Vries, H., et al. (2017). Modeling longitudinal relationships between habit and physical activity: Two crosslagged panel design studies in older adults. J. Aging Phys. Act., 25, 464-473.

van Bree, R.J.H., van Stralen, M.M., Mudde, A.N., Bolman, C., de Vries, H., \& Lechner, L. (2015). Habit as mediator of the relationship between prior and later physical activity: A longitudinal study in older adults. Psychol. Sport Exerc., 19, 95-102.

Wood, W. (2017). Habit in personality and social psychology. Pers. Soc. Psychol. Rev., 21, 389-403.

Wood, W., Labrecque, J., Lin, P.-Y., \& Ruenger, D. (2014). Habits in dual process models. In J. Sherman, B. Gawronski, \& Y. Trope (Eds.), Dual process theories of the social mind pp. 371385). New York, NY: Guildford. 
Table 1

Standardized Path Coefficients for Direct and Indirect Effects for the Meta-Analytic Structural Equation Model (Stage 2) of the Reasoned Action Approach Excluding and Including Past Behavior with Model Comparisons

\begin{tabular}{|c|c|c|c|c|c|c|c|c|c|c|c|}
\hline \multirow[t]{3}{*}{ Effect } & \multicolumn{3}{|c|}{$\begin{array}{c}\text { Model excluding past } \\
\text { behavior }\end{array}$} & \multicolumn{3}{|c|}{$\begin{array}{l}\text { Model including past } \\
\text { behavior }\end{array}$} & \multicolumn{5}{|c|}{ Model comparisons } \\
\hline & \multirow[t]{2}{*}{$\beta$} & \multicolumn{2}{|c|}{ LB CI95 } & \multirow[t]{2}{*}{$\beta$} & \multicolumn{2}{|c|}{$\mathrm{LB} \mathrm{CI}_{95}$} & \multirow[t]{2}{*}{$\beta_{\text {diff }}{ }^{\mathrm{a}}$} & \multicolumn{2}{|c|}{$\mathrm{CI}_{95}$} & \multirow[t]{2}{*}{$t^{\mathrm{b}}$} & \multirow[t]{2}{*}{$p$} \\
\hline & & LL & UL & & LL & UL & & LL & UL & & \\
\hline \multicolumn{12}{|l|}{ Direct effects } \\
\hline Experiential attitude $\rightarrow$ Intention & .296 & .244 & .347 & .245 & .188 & .299 & .052 & -.024 & .127 & 1.339 & .181 \\
\hline Instrumental attitude $\rightarrow$ Intention & .092 & .033 & .150 & .072 & .009 & .135 & .020 & -.066 & .106 & 0.457 & .648 \\
\hline Injunctive norm $\rightarrow$ Intention & .079 & .037 & .119 & .071 & .030 & .111 & .008 & -.049 & .065 & 0.267 & .790 \\
\hline Descriptive norm $\rightarrow$ Intention & .151 & .099 & .202 & .090 & .034 & .144 & .061 & -.014 & .136 & 1.593 & .111 \\
\hline Autonomy $\rightarrow$ Intention & .032 & -.041 & .101 & .022 & -.054 & .094 & .010 & -.092 & .112 & 0.199 & .842 \\
\hline Capacity $\rightarrow$ Intention & .318 & .254 & .383 & .267 & .197 & .335 & .051 & -.043 & .145 & 1.056 & .291 \\
\hline Autonomy $\rightarrow$ Behavior & .034 & -.031 & .098 & .014 & -.067 & .092 & .021 & -.080 & .122 & 0.401 & 689 \\
\hline Capacity $\rightarrow$ Behavior & .106 & .033 & .176 & .074 & -.010 & .156 & .032 & -.076 & .140 & 0.574 & .566 \\
\hline Experiential attitude $\rightarrow$ Behavior & .064 & .017 & .108 & .024 & -.032 & .077 & .040 & -.031 & .111 & 1.095 & .273 \\
\hline Intention $\rightarrow$ Behavior & .370 & .304 & .436 & .175 & .075 & .265 & .195 & .081 & .310 & 3.316 & .001 \\
\hline $\mathrm{PB} \rightarrow$ Experiential attitude & - & - & - & .401 & .353 & .450 & - & - & - & - & - \\
\hline $\mathrm{PB} \rightarrow$ Instrumental attitude & - & - & - & .290 & .229 & .350 & - & - & - & - & - \\
\hline $\mathrm{PB} \rightarrow$ Injunctive norm & - & - & - & .265 & .230 & .300 & - & - & - & - & - \\
\hline $\mathrm{PB} \rightarrow$ Descriptive norm & - & - & - & .338 & .285 & .391 & - & - & - & - & - \\
\hline $\mathrm{PB} \rightarrow$ Autonomy & - & - & - & .224 & .124 & .325 & - & - & - & - & - \\
\hline $\mathrm{PB} \rightarrow$ Capacity & - & - & - & .398 & .333 & .462 & - & - & - & - & - \\
\hline $\mathrm{PB} \rightarrow$ Intention & - & - & - & .272 & .197 & .344 & - & - & - & - & - \\
\hline $\mathrm{PB} \rightarrow$ Behavior & - & - & - & .412 & .317 & .509 & - & - & - & - & - \\
\hline \multicolumn{12}{|l|}{ Indirect effects } \\
\hline Experiential attitude $\rightarrow$ Intention $\rightarrow$ Behavior & .110 & .085 & .138 & .043 & .018 & .070 & .067 & .030 & .104 & 3.516 & .000 \\
\hline Instrumental attitude $\rightarrow$ Intention $\rightarrow$ Behavior & .034 & .012 & .057 & .013 & .001 & .029 & .022 & -.005 & .048 & 1.596 & .110 \\
\hline
\end{tabular}


Injunctive norm $\rightarrow$ Intention $\rightarrow$ Behavior

Descriptive norm $\rightarrow$ Intention $\rightarrow$ Behavior

Autonomy $\rightarrow$ Intention $\rightarrow$ Behavior

Capacity $\rightarrow$ Intention $\rightarrow$ Behavior

$\mathrm{PB} \rightarrow$ Experiential attitude $\rightarrow$ Intention $\rightarrow$ Behavior

$\mathrm{PB} \rightarrow$ Instrumental attitude $\rightarrow$ Intention $\rightarrow$ Behavior

$\mathrm{PB} \rightarrow$ Injunctive norm $\rightarrow$ Intention $\rightarrow$ Behavior

$\mathrm{PB} \rightarrow$ Descriptive norm $\rightarrow$ Intention $\rightarrow$ Behavior

$\mathrm{PB} \rightarrow$ Autonomy $\rightarrow$ Intention $\rightarrow$ Behavior

$\mathrm{PB} \rightarrow$ Capacity $\rightarrow$ Intention $\rightarrow$ Behavior

$\mathrm{PB} \rightarrow$ Autonomy $\rightarrow$ Behavior

$\mathrm{PB} \rightarrow$ Capacity $\rightarrow$ Behavior

$\mathrm{PB} \rightarrow$ Experiential attitude $\rightarrow$ Behavior

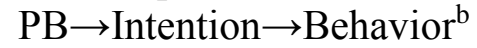

Total effects

Autonomy $\rightarrow$ Behavior $^{\mathrm{c}}$

Capacity $\rightarrow$ Behavior $^{\mathrm{c}}$

Experiential attitude $\rightarrow$ Behavior $^{\mathrm{c}}$

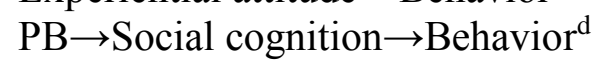

$\mathrm{PB} \rightarrow$ Behavior $^{\mathrm{C}}$

Correlations $^{\mathrm{e}}$

Experiential attitude $\leftrightarrow$ Instrumental attitude

Experiential attitude $\leftrightarrow$ Injunctive norm

Experiential attitude $\leftrightarrow$ Descriptive norm

Experiential attitude $\leftrightarrow$ Autonomy

Experiential attitude $\leftrightarrow$ Capacity

Instrumental attitude $\leftrightarrow$ Injunctive norm

Instrumental attitude $\leftrightarrow$ Descriptive norm

Instrumental attitude $\leftrightarrow$ Autonomy

Instrumental attitude $\leftrightarrow$ Capacity

Injunctive norm $\leftrightarrow$ Descriptive norm

$\begin{array}{ccc}.029 & .014 & .045 \\ .056 & .035 & .080 \\ .012 & -.015 & .038 \\ .118 & .088 & .154 \\ - & - & - \\ - & - & - \\ - & - & - \\ - & - & - \\ - & - & - \\ - & - & - \\ - & - & - \\ - & - & - \\ - & - & - \\ - & - & -\end{array}$

\section{.012}

.016

.004

.047

.017

.004

.003

.005

.001

.019

.003

.029

.010

.047

\section{.004}

.005

.024

$-.010$

.020

.007

.000

.001

.002

$-.003$

.008

$-.020$

$-.004$

$-.014$

.023

.032

.018

.077

.028

.008

.006

.011

.004

.032

.017

.058

.029

.072

$\begin{array}{rrr}.017 & -.002 & .035 \\ .040 & .014 & .066\end{array}$

1.762

2.972

$\begin{array}{lll}.008 & -.021 & .037\end{array}$

$\begin{array}{lll}.067 & .030 \quad .104\end{array}$

0.521

.602

$-$

$$
-
$$

$$
\text { - }
$$

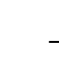$$
-
$$

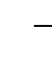

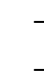$$
-
$$

$\begin{array}{lll}.046 & -.017 & .107\end{array}$

.017

$-.067$

.099$$
.029
$$

.103

.107

-.074
.007

.007

.131

.200

.011

.117

.067
.138

.098

.177

.613
0.548

2.074

3.193

.584

.038

.001$$
-
$$$$
.551
$$

.488

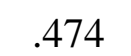

\section{.333}

\section{.282}

.229

.453

.382

.221

.229

.340

.386
.436
.304
.253
.181
.410
.350
.177
.182
.299
.338

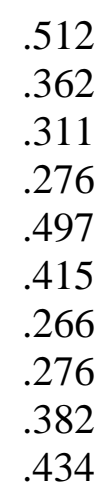

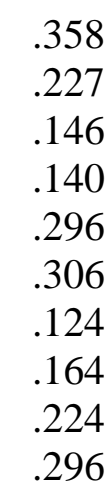

$\begin{array}{ll}.309 & .405 \\ .192 & .261 \\ .108 & .184 \\ .077 & .201 \\ .242 & .349 \\ .268 & .343 \\ .073 & .174 \\ .105 & .220 \\ .171 & .276 \\ .245 & .347\end{array}$

.116
.106
.136
.089
.157
.076
.098
.065
.116
.089

.055
.061
.088
.011
.088
.026
.030
-.009
.049
.019

\subsection{0}

\subsection{3}

5.595

2.232

4.456

3.001

2.837

1.731

3.391

2.499
.000

.000

.000

.026

.000

.003

.005

.084

.001

.012 


\begin{tabular}{|c|c|c|c|c|c|c|c|c|c|c|c|}
\hline Injunctive norm $\leftrightarrow$ Autonomy & .178 & .140 & .216 & .118 & .071 & .165 & .060 & .000 & .120 & 1.948 & .051 \\
\hline Injunctive norm $\leftrightarrow$ Capacity & .292 & .256 & .329 & .186 & .142 & .229 & .107 & .050 & .163 & 3.704 & .000 \\
\hline Descriptive norm $\leftrightarrow$ Autonomy & .160 & .110 & .209 & .082 & .019 & .144 & .077 & -.002 & .157 & 1.903 & .057 \\
\hline Descriptive norm $\leftrightarrow$ Capacity & .245 & .169 & .320 & .097 & .015 & .180 & .148 & .036 & .259 & 2.589 & .010 \\
\hline Autonomy $\leftrightarrow$ Capacity & .390 & .320 & .460 & .303 & .221 & .383 & .087 & -.020 & .194 & 1.597 & .110 \\
\hline
\end{tabular}

Note. $\beta$ = Standardized path coefficient; LB CI 95 = Likelihood based 95\% confidence interval; LL = Lower limit of CI 95 ; UL = Upper limit of

$\mathrm{CI}_{95} ; \mathrm{CI}_{95}=$ Conventional $95 \%$ confidence interval; $\beta_{\text {diff }}=$ Difference in standardized path coefficient; $\mathrm{PB}=$ Past behavior; ${ }^{\mathrm{a}} \mathrm{Model}$ comparisons made using Schenker and Gentleman's (2001) 'standard method' using confidence intervals about the mean difference derived from Wald standard errors; ' ${ }^{\mathrm{b}}$ Indirect effect of past behavior on behavior through intention only; ${ }^{\mathrm{c}}$ Total effect including direct and indirect effects; ${ }^{\mathrm{d}}$ Total indirect effect of past behavior on behavior excluding direct effect; ${ }^{\circ}$ Correlations among residuals of the predictors of intention, decreases in the size of these correlations with the inclusion of past behavior indicates that shared variance in the residuals of the constructs is accounted for by past behavior. 
Figure 1. Proposed model tested in path analysis of meta-analytic correlations of the reasoned action approach for health behavior excluding past behavior.

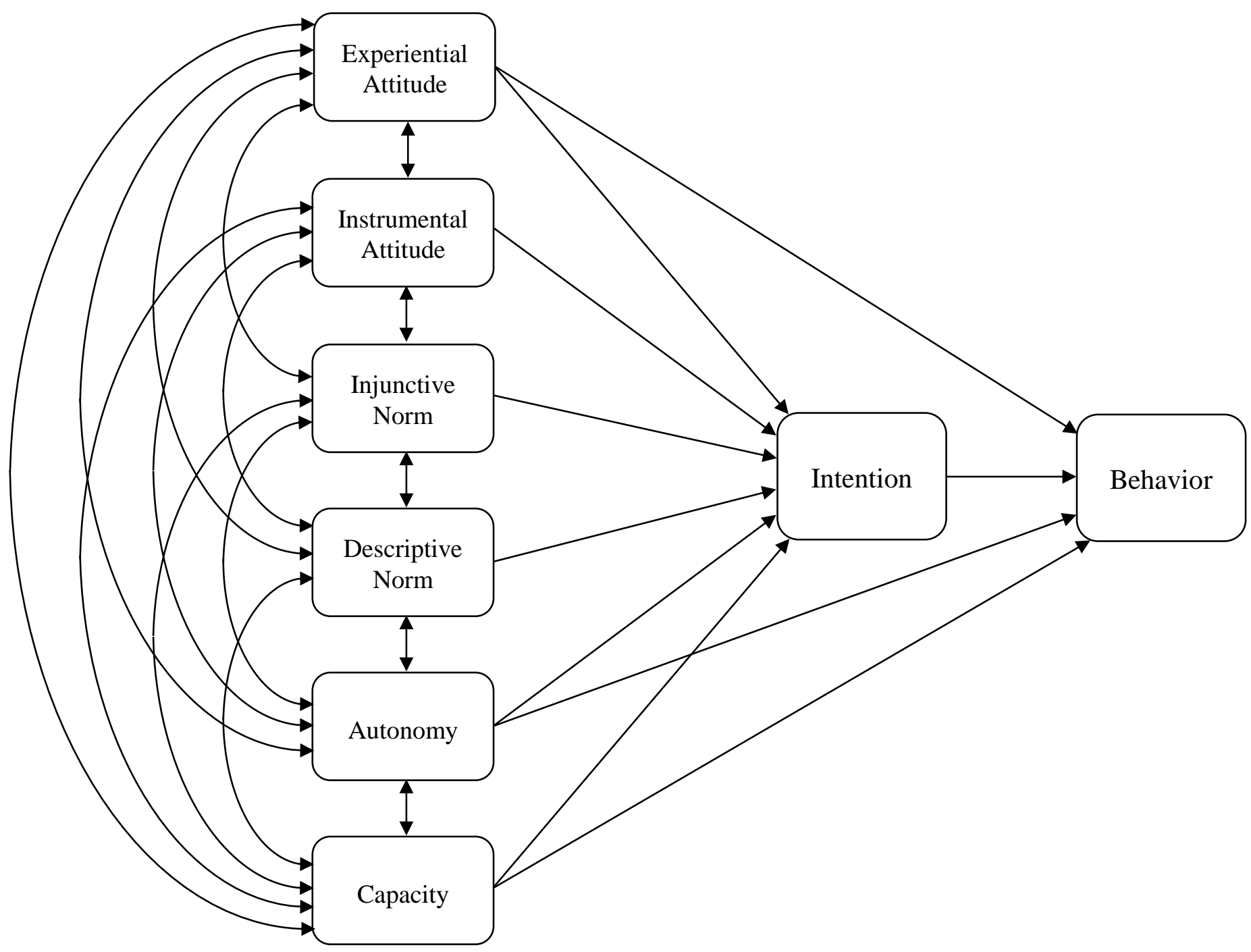


Figure 2. Proposed model tested in path analysis of meta-analytic correlations of the reasoned action approach for health behavior including past behavior.

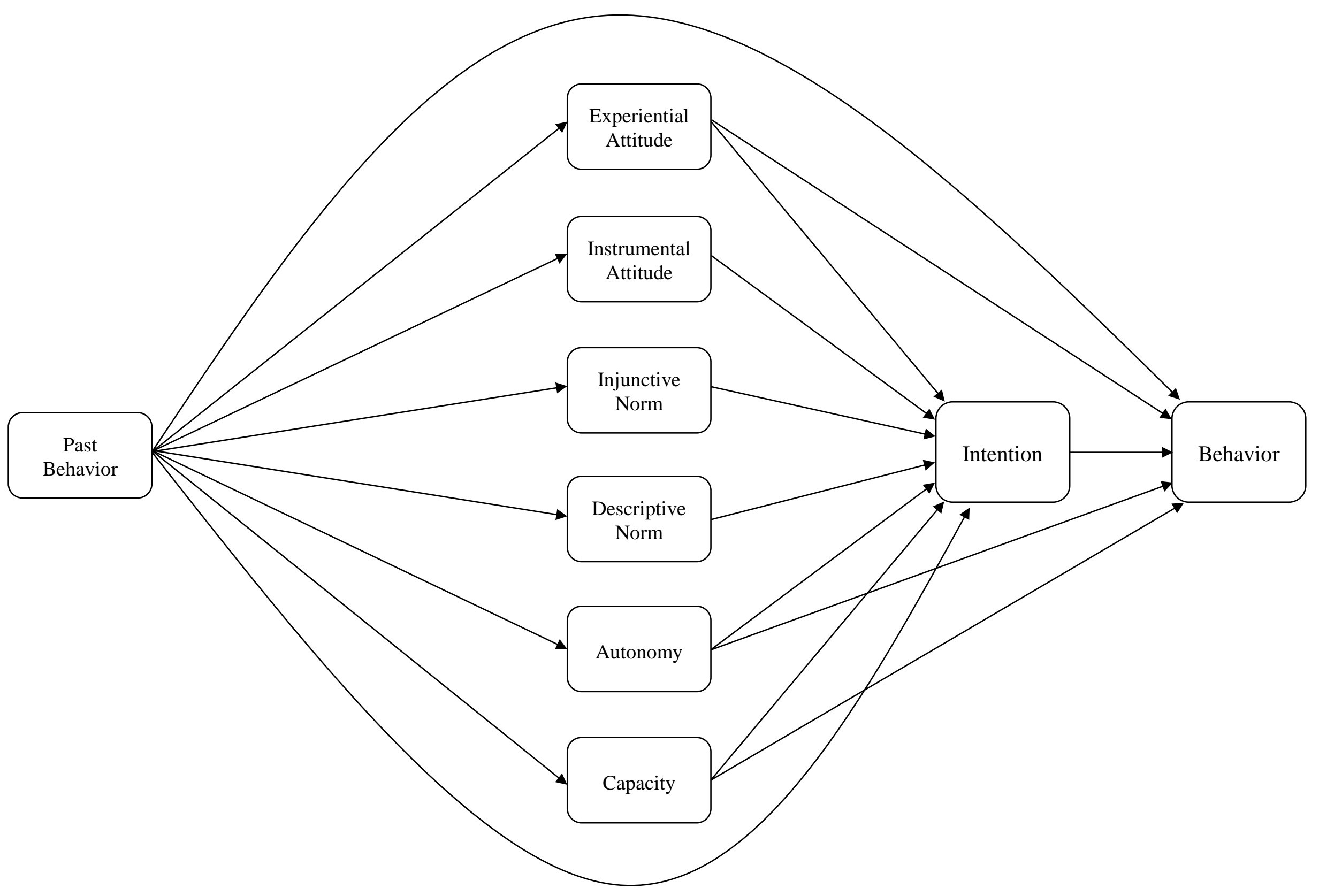

